\title{
Effect of Treatment by Hyaluronic Acid (HA) In Hip Osteoartrithis, Executed by Eco Tomografy Guide and Gait Analysis
}

\author{
S Boccaccio*, A Zocco and S Denaro \\ Department of Rehabilitation, Hospital Rizza, Siracusa, Italy \\ *Corresponding author: S Boccaccio, Department of Rehabilitation, Hospital Rizza, Siracusa, Italy
}

\begin{tabular}{|c|c|}
\hline ARTICLE INFO & ABSTRACT \\
\hline Received: 蔧 August 31, 2020 & Citation: S Boccaccio, A Zocco, S Denaro. Effect of Treatment by Hyaluronic Acid (HA) In \\
\hline Published: September 10, 2020 & $\begin{array}{l}\text { Hip Osteoartrithis, Executed by Eco Tomografy Guide and Gait Analysis. Biomed J Sci \& } \\
\text { Tech Res 30(2)-2020. BJSTR. MS.ID. } 004930 \text {. }\end{array}$ \\
\hline
\end{tabular}

\section{Introduction}

Osteoarthritis is a multi-factorial factor disease, as obesity, age, genetic factors, metabolic or inflammation diseases. In Italy, five million of patients are suffering from symptomatic forms with pain and reduction of mobility. The treatment lead to pain reduction and in increasing of articular movement, with analgesic, steroidal and non-steroidal drugs, and physiotherapist treatments [1]. Hip osteoarthritis represents 7.7 per cent of OA localization, and is characterized by pain and impaired movement. Intra-articular joint infiltrations of hyaluronic acid (HA), using Eco tomography guide is new possible treatment [2]. The use of HA to restore the biologic properties sinovial fluid in the joint, is known. It was widely used in several forms of $\mathrm{OA}$, as knee [3] or shoulder. In hip osteoarthritis an echographyguide is used to have an optimal control of the needle positioning in the joint [4]. In literature several studied investigate the effect of the treatment on hip $\mathrm{OA}$, but few correlates to gait quality [5].

\section{The Aim}

The aim of our study evaluated the effect of the treatment with Hyaluronic acid (HA), executed by Eco tomography guide, in patients with hip osteoartrithis, using functionals scale (vas, lequesne index), correlating to quality gait.

\section{Methods}

From January to May we have treated 13 patients with hip osteoarthritis by intra-articular joint infiltrations, of high molecular weight hyaluronic acid, executed by Eco tomography guide. 3 patients dropped out for bad compliance to treatment.

The characteristics of patients were (Table 1):

- 6 male

- 7 female, Mean age 67 (dev. Stand. 13,79)

- 5 patients with $\mathrm{OA}$ on the right side

- 6 patients with OA on the left side

- $\quad 2$ bilateral Inclusion and exclusion criteria of our study

Table 1: The characteristics of patients were

\begin{tabular}{|c|c|c|}
\hline Gender & Age & Side \\
\hline m & 39 & sx \\
\hline m & 76 & sx \\
\hline m & 82 & sx \\
\hline f & 48 & $\mathrm{dx}$ \\
\hline $\mathrm{f}$ & 60 & $\mathrm{dx}$ \\
\hline $\mathrm{m}$ & 80 & $\mathrm{bil}$ \\
\hline $\mathrm{m}$ & 65 & $\mathrm{dx}$ \\
\hline $\mathrm{m}$ & 54 & $\mathrm{dx}$ \\
\hline $\mathrm{f}$ & 80 & $\mathrm{dx}$ \\
\hline $\mathrm{f}$ & 79 & $\mathrm{bil}$ \\
\hline $\mathrm{f}$ & 61 & $\mathrm{sx}$ \\
\hline $\mathrm{f}$ & 69 & $\mathrm{dx}$ \\
\hline $\mathrm{f}$ & 78 & $\mathrm{sx}$ \\
\hline $\mathrm{f}$ & & \\
\hline
\end{tabular}




\section{Inclusions Criteria}

- Hip osteoarthritis defined by radiologic grading (Kellgrenn and Lawrence scale [6]), II-III age between 30 and 80 years, no pharmacological treatment, good compliance to treatment, with total adherence to steps study.

\section{Exclusions Criteria}

- $\quad$ Bad compliance to treatment, presence of other hip pathologies as head hip necrosis, tumor, or candidates to hip replacement, dysplasia or congenital abnormality, contraindications to intra-articular hyaluronic-acid preparations, skin or infections disease, systemic or intra-articular corticosteroid injections in the last 6 months, anticoagulant therapy.

Patients are treated by three (one a week) injection of HA (from 1200 to $2000 \mathrm{~K}$-dalton) and submitted to evaluation by:

- $\quad$ Lequesne scale for functional

- V.A.S. (visuo-analogic scale), for pain

Hyaluronan (also called hyaluronic acid or hyaluronate or HA) is an anionic non-sulfated glycosaminoglycan distributed in several tissues, as connective, epithelial, neural. The use of Hyaluronic acid on osteoarthritis is noted. HA is a component of the synovial fluid, have a protective effect on cartilage, increasing the viscosity and reducing the production of chemicals inflammation mediator. Polymers of hyaluronan can range in size from 5,000 to 20,000,000, for hip injections we used polymers at high molecular weight. Moreover, in order to correlate the functional effect of treatment to treatment, we have used a gait analysis system called BTS. BTS G-WALK is a wireless system composed by triaxial accelerometer, a magnetic sensor, and a triaxial gyroscope. Positioned on L5 the system provides data of gait analysis as cadence, velocity, single support, and others.

In our study the parameters of gait considered are:

- Speed as evaluation of functional recovery

- $\quad$ Single support as evaluation of pain during gait

- Stride length as evaluation of hip extension improvement

- Gait length as evaluation of hip extension recovery

Evaluations have been made at:

- $\quad$ T0, before first treatment

- $\quad \mathrm{T} 1$, at 4 weeks

- $\quad \mathrm{T} 2$, at 8 weeks

Also, we have evaluated the patients by Kellgren Lawrence Grading Scale (Table 2), a useful and relatively straight forward grading system for X-rays of the arthritic hip. Their system considers:
Table 2.

\begin{tabular}{|c|c|}
\hline Gender & Grading \\
\hline m & 23 \\
\hline m & 3 \\
\hline m & 3 \\
\hline f & 2 \\
\hline f & 3 \\
\hline m & $2 / \mathrm{bil}$ \\
\hline m & 3 \\
\hline m & 3 \\
\hline f & 2 \\
\hline f & $2 \mathrm{dx} / 3 \mathrm{sx}$ \\
\hline f & 3 \\
\hline f & 3 \\
\hline f & \\
\hline
\end{tabular}

1. Joint space narrowing

2. Osteophytic lipping

3. Sclerosis

4. Bone contour deformity

Our patient included by treatment were between II and III radiological grades of scale.

\section{Method of Infiltrations}

Normally, the intra-articular infiltration is carried out by a blind technique. Hip is a depth joint, and the use of blind technique can present some difficulties. According to literature, we used ecotomografy guide using a linear probe of 7,5-10 Mhz, equipped by a guide support for spinal needle, and guide trace on echography screen, to have an optimal control of needle into the joint (8).

\section{Statistical Analysis}

All analyses were performed using statistical program. We used the paired $t$ test in order to compare the data. We have studied the statistical difference at T0, T1 and T2, comparing the evolution of Lequesne Index, V.A.S. and the parameters of gait analysis considered.

\section{Results}

All results showed a statistical increase of parameters considered. Lequesne Index showed a mean variation from 7.41 to $2.75, \mathrm{p}<0,01$ (Table 3 ), and that indicates an improvement of functional state. This scale has developed for evaluation of severity hip osteoarthritis and can be used to assess the effectiveness of therapeutic interventions. Content a composite measure aggregating symptoms and function, which are not graded separately, where pain is analyzed by 5 items, maximum distance walked by 2 items, and activities of daily living (ADL) by 4 items. The results showed a decrease of Lequesne Index, mean value at T0 
7.41, mean value at T2 2.25, p 0,01 Data shows a decrease of value to V.A.S, from mean value 4.72 at T0, to 1 at T2, p 0,01 (Table 4). The results showed a decrease of Lequesne Index, mean value at T0 7.41, mean value at T2 2.25, $\mathrm{p}<0,01$ Data shows a decrease of value to V.A.S, from mean value 4.72 at $\mathrm{T} 0$, to 1 at $\mathrm{T} 2, \mathrm{p}<0,01$ (Figures 1-4) The parameters of gait showed an improvement of value, the diagram shows the value at To and T2. Gait parameter considered showed a mean value of speed at T0 56,33, at T2 58,38,; average value of the percentage ratio of the step length of the subject at T0 $=69,2$, at $\mathrm{T} 2=72,6, \mathrm{p}<0,01$.

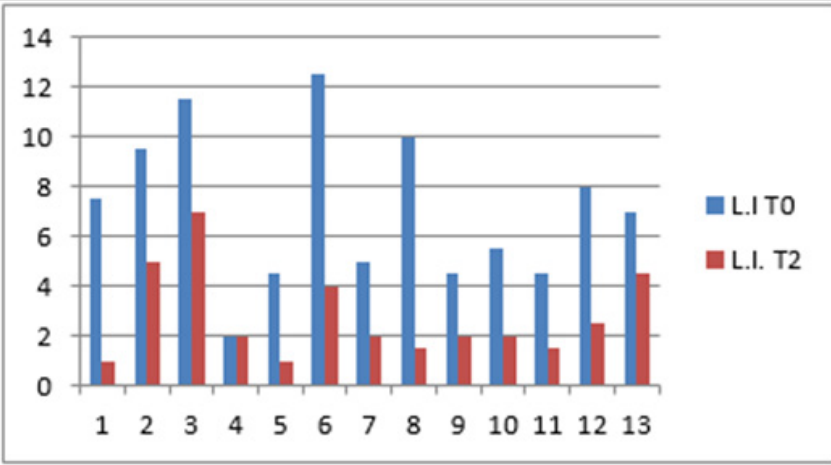

Figure 1.

Table 3.

\begin{tabular}{|c|c|c|}
\hline Gender & L.I. I & L.I. II \\
\hline m & 7.5 & 1 \\
\hline m & 9.5 & 5 \\
\hline m & 11.5 & 7 \\
\hline f & 2 & 2 \\
\hline f & 4.5 & 1 \\
\hline m & 12,5 & 4 \\
\hline m & 5 & 2 \\
\hline m & 10 & 1,5 \\
\hline f & 4.5 & 2 \\
\hline f & 5.5 & 2 \\
\hline f & 4.5 & 1.5 \\
\hline f & 8 & 2.5 \\
\hline f & 7 & 4.5 \\
\hline
\end{tabular}

Table 4.

\begin{tabular}{|c|c|c|c|c|}
\hline Gender & Speed T0 & Lenght T0 & Speed T2 & Lenght T2 \\
\hline m & 50,8 & 75,8 & 57,7 & 83,1 \\
\hline m & 47,9 & 72,3 & 57,1 & 74,2 \\
\hline m & 47,9 & 58,5 & 50,8 & 62,6 \\
\hline f & 53,1 & 72,2 & 56,2 & 72 \\
\hline f & 56 & 67,6 & 68,7 & 73,1 \\
\hline
\end{tabular}

\begin{tabular}{|c|c|c|c|c|}
\hline $\mathrm{m}$ & 61 & 74,1 & 69,9 & 80,6 \\
\hline $\mathrm{m}$ & 61,9 & 67,7 & 74,7 & 76,2 \\
\hline $\mathrm{m}$ & 63,2 & 81,3 & 61 & 73,6 \\
\hline $\mathrm{f}$ & 47,5 & 57,9 & 49,8 & 63 \\
\hline $\mathrm{f}$ & 52,6 & 69,3 & 49,9 & 68,7 \\
\hline $\mathrm{f}$ & 52 & 62,5 & 61,1 & 63,8 \\
\hline $\mathrm{f}$ & 57,3 & 76,5 & 49,5 & 74,9 \\
\hline $\mathrm{f}$ & 44,3 & 66,3 & 39,5 & 68 \\
\hline
\end{tabular}

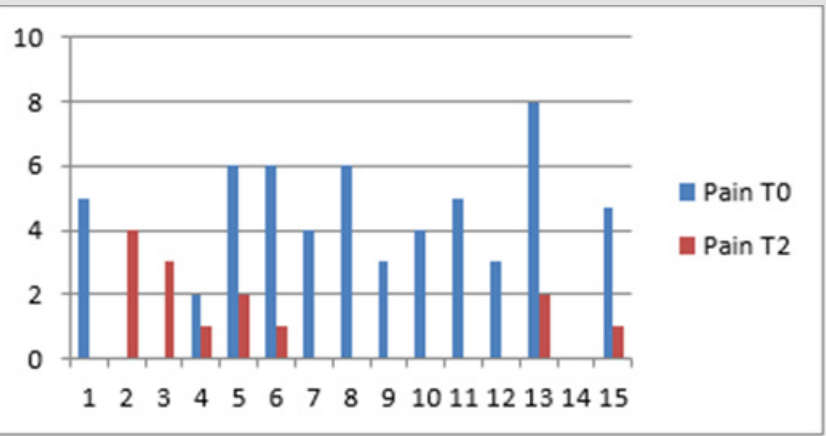

Figure 2.

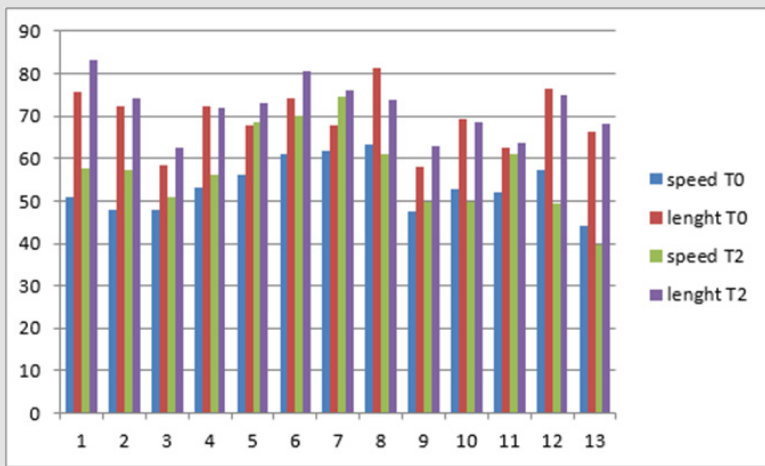

Figure 3.

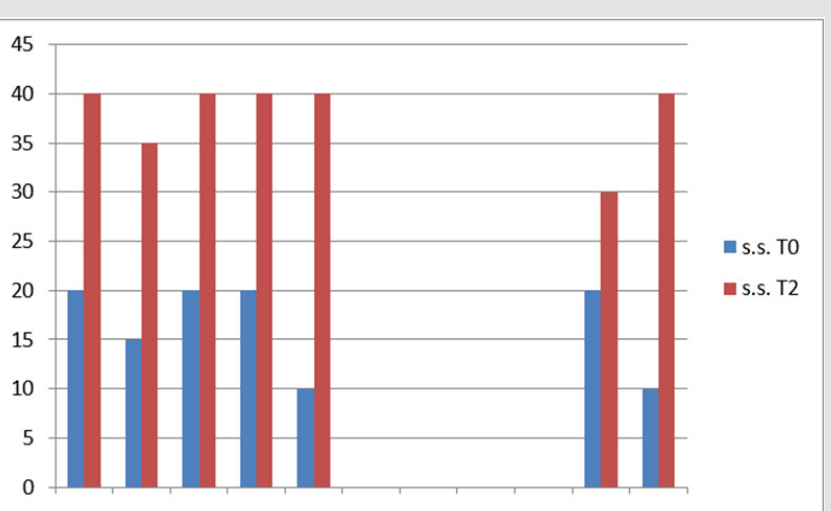

Figure 4. 


\section{Discussion}

Pain and decrease of articular movement as flexo-extension and rotation characterize hip osteoarthritis, this reduced gait quality. Several studies investigate hip articular capacity after and before treatments as, pharmacological or physiotherapist, but few investigate gait performances.In our group of patients with hip osteoarthritis defined by K.L. scale, between first and third grade, several treatments have been possible. Our use of joint infiltration of HA by eco-tomography guide, have obtained two results: pain relief, evaluated by improvement of mean value of V.A.S. $(p<0,01)$, and increase of functional hip capacity, evaluated by improvement of Lequesne Index $(p<0,01)$.Gait analysis we allowed us to correlate these results. In particular, the increase of stride to hip flexoexention $(\mathrm{p}<0,01)$; speed $(\mathrm{p}<0.01)$ to Lequesne Index, single support phase, to V.A.S. $(\mathrm{p}<0,01)$. We think that this last parameter it is important, with an increment in 7 subjects $(20$ per cent of mean). This it could indicate a change of gait scheme, with an improvement of articular performance. It is during this phase that the patient feels the pain and reduce the articular stress.

\section{Conclusion}

Our study confirms the intra-articular joint infiltrations of hyaluronic acid, executed by Eco tomography guide, as a possible treatment to reduce the pain and increase the mobility in patients affected by hip osteoarthritis. Gait analysis can be a new method for evaluation of this patients.

\section{References}

1. Qvistgaard E, Christensen R, Torp Pedersen S, Bliddal H (2006) Intraarticular treatment of hip osteoarthritis: A randomized trial of hyaluronic acid, corticosteroid, and isotonic saline. Osteoarthritis Cartilage 14(2): 163-170.

2. Migliore A, Tormenta S, Martin LS, Valente C, Massafra U, et al. (2005) Open pilot study of ultrasound-guided intra-articular injection of hylan G-F 20 (Synvisc) in the treatment of symptomatic hip osteoarthritis. Clin Rheumatol 24(3): 285-289.

3. Bellamy N, Campbell J, Robinson V, Gee T, Bourne R, et al. (2006) Viscosupplementation for the treatment of osteoarthritis of the knee. Cochrane Database Syst Rev 2: CD005321.

4. Migliore A, Martin LS, Alimonti A, Valente C, Tormenta S (2003) Efficacy, and safety of viscosupplementation by ultrasound-guided intra-articular injection in osteoarthritis of the hip. Osteoarthritis Cartilage 11(4): 305306.

5. Paoloni M, Di Sante L, Dimaggio M, Bernetti A, Mangone M, et al. (2012) Kinematic and kinetic modifications in walking pattern of hip osteoarthritis patients induced by intra-articular injections of hyaluronic acid. Clin Biomech 27(7): 661-665.

6. Kelgrenn JH, Lawrence JS (1957) Radiological Assessment of osteoarthosis. Ann Reum Dis 16: 494-502.

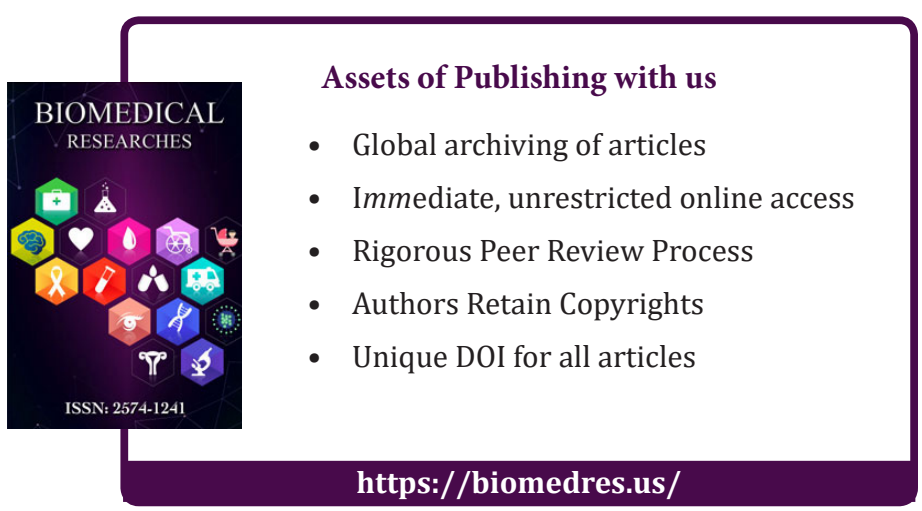

